\title{
Seltene Erkrankungen in der Pneumologie und damit verbundene Herausforderungen
}

\author{
Rare Diseases in Pulmonary Medicine and its Challenges
}

Autoren

Institute
M. Wencker ${ }^{1}$, H. Teschler ${ }^{1}$, C. Vogelmeier ${ }^{2}$, R. Koczulla ${ }^{2}$

${ }^{1}$ Ruhrlandklinik, Westdeutsches Lungenzentrum, Abteilung Pneumologie, Universitätsklinikum Essen (Chefarzt Prof. Dr. H. Teschler)

${ }^{2}$ Universitätsklinikum Gießen und Marburg, Standort Marburg, Klinik für Innere Medizin mit Schwerpunkt Pneumologie (Direktor Prof. Dr. C. Vogelmeier) eingereicht 16.3.2012 akzeptiert nach Revision 18. 4. 2012

\section{Bibliografie}

Dol http://dx.doi.org/ 10.1055/s-0032-1309876

Online-Publikation: 12.6.2012

Pneumologie 2012; 66: 437-441

(c) Georg Thieme Verlag KG

Stuttgart · New York

ISSN 0934-8387

Korrespondenzadresse

Dr. med. Marion Wencker Speicherstr. 9

60327 Frankfurt

mwencker@arcor.de

\section{Zusammenfassung \\ $\nabla$}

Die medizinische Unter- und Fehlversorgung von Patienten mit seltenen Erkrankungen ist von allen Akteuren im Gesundheitswesen erkannt. Es werden große Anstrengungen unternommen, um das Wissen über die Krankheiten und die Versorgung betroffener Patienten zu verbessern.

Politische Initiativen auf europäischer Ebene haben die Verbesserung von Versorgungsstrukturen für die Betreuung von Patienten mit seltenen Erkrankungen in den Mitgliedsländern zum Ziel. Die geschaffenen Anreize für die Entwicklung von Medikamenten für seltene Erkrankungen haben zu vermehrten Studienaktivitäten und Zulassungen von Orphan Drugs geführt.

Patienten sind national wie auch international in verschiedenen Patientenorganisationen für ihre jeweilige Erkrankung sowie in Dachverbänden organisiert.

Viele Experten engagieren sich in nationalen und internationalen Netzwerken und Registern, die Forschungsdaten für seltene Erkrankungen in hoher Qualität generieren und publizieren.

Die Suche nach spezialisierten Partnern für Fragen zu einer bestimmten seltenen Lungenerkrankung wird durch die vorhandene Infrastruktur erleichtert.

\section{Einleitung \\ $\nabla$}

Nach der in Europa gültigen Definition ist eine Erkrankung „selten“, wenn weniger als einer von 2000 Menschen unter einem spezifischen Krankheitsbild leidet. Seltene Erkrankungen stellen eine besondere Herausforderung für Patienten, Ärzte und das Gesundheitssystem dar. Dies gilt zunächst für die korrekte und zeitnahe Diagnosestellung aber auch für die nachfolgende weitere medizinische Betreuung der Betroffenen. Der Begriff „seltene Erkrankung“ impliziert schon, dass die genannte Krankheit nur eine untergeordnete

\section{Abstract \\ $\nabla$}

The importance of rare disease is appreciated by all parties and tremendous effort is made to increase the knowledge about the individual disorders and improve the care of affected patients. Political initiatives on a European level aim to improve the structure of medical care for patients with rare diseases in each member state.

The provided incentives for the development of medicines for orphan diseases have led to increased research activities and numbers of licensed Orphan Drugs.

Patients are organized nationally and internationally in various patient organizations and umbrella organizations. They are involved in health care policy, support the detection and research of rare diseases and offer support to affected patients and families with educational meetings and materials as well as options for discussions. Many experts are engaged in national and international networks and registries that generate and publish high quality research data on rare diseases.

A well developed infrastructure is in place to support the search for qualified partners that can be of assistance with specific questions in a rare lung disease.

Rolle in der täglichen medizinischen Praxis spielt. Ein Allgemeinarzt, aber auch ein Fach- oder Klinikarzt betreuen oft keinen oder nur einzelne Patienten mit einer solchen Erkrankung. Das entsprechende Wissen über die Diagnosestellung, mögliche Komplikationen und die vorhandenen Therapiemöglichkeiten ist daher oft nur rudimentär vorhanden.

Nach Schätzungen der Europäischen Union (EU) gibt es zwischen 5000 und 8000 seltene Erkrankungen, die etwa 6 bis $8 \%$ der Bevölkerung im Laufe ihres Lebens betreffen [1]. Dies entspricht etwa 4 Millionen in Deutschland. Zum Vergleich: 


\begin{tabular}{|c|c|c|c|}
\hline & $\begin{array}{l}\text { Häufigkeit } \\
\text { pro } 100,000^{1}\end{array}$ & ICD 10 & $\mathrm{MIM}^{2}$ \\
\hline Idiopathische Lungenfibrose & 27 & J 84.1 & 178500 \\
\hline Alpha1-Antitrypsin-Mangel (AATM) & 25 & E 88.0 & 613490 \\
\hline Sarkoidose & 15 & D 86 & 181000 \\
\hline Bronchopulmonale Dysplasie & 13 & P 27.1 & - \\
\hline Zystische Fibrose & 12 & E 84 & 219700 \\
\hline Kleinzelliges Bronchial-Ca & 10 & C34.9 & 182280 \\
\hline Wegener Granulomatose & 6,6 & M31.3 & 608710 \\
\hline Amyotrophe Lateralsklerose & 5,2 & G12.2 & 105400 \\
\hline Primäre ziliäre Dyskinesie & 5 & - & 244400 \\
\hline Akute interstitielle Pneumonie (AIP) & 3,8 & - & - \\
\hline Hereditäre hämorrhagische Teleangiektasie (M. Osler) & 3,5 & 178.0 & 187300 \\
\hline Akute Graft- versus Host-Erkrankung & 3,4 & - & - \\
\hline Mesotheliom & 2,7 & C45 & 156240 \\
\hline Exogen allergische Alveolitis (EAA) & $2,5[21]$ & 67.0 & 145300 \\
\hline Behcet-Syndrom & 2,5 & M 35.2 & 109650 \\
\hline Undine-Syndrom & 2,25 & G47.3 & 209880 \\
\hline Langerhans-Zell-Histiozytose (Histiocytosis X) & 2 & D 76.0 & 604856 \\
\hline Birth-Hogg-Dubé-Syndrom & 2 & - & 135150 \\
\hline Pulmonale arterielle Hypertonie (PAH) & 1,5 & 127.0 & 178600 \\
\hline Churg-Strauss-Syndrom & 1 & M 30.1 & - \\
\hline Hermansky-Pudlak-Syndrom & 0,15 & E 70.3 & 203300 \\
\hline LAM & 0,1 & 189.8 & 606690 \\
\hline Pulmonale Alveolarproteinose & 0,1 & - & 265120 \\
\hline Panbronchilitis & $<0,1$ & - & 604809 \\
\hline Idiopathische pulmonale Hämosiderose & 250 Fälle & & 178550 \\
\hline Akute eosinophile Pneumonie & >100 Fälle & J 82 & - \\
\hline Lymphoide interstitielle Pneumonie & unbekannt & - & 247610 \\
\hline Bronchiolitis obliterans mit organisierender Pneumonie (BOOP) & unbekannt & 84.8 & - \\
\hline Desquamative interstitielle Pneumonie (DIP) & unbekannt & - & 263000 \\
\hline Alveolares Hämorrhagiesyndrom & unbekannt & J 98.8 & - \\
\hline
\end{tabular}

Tab. 1 Liste der seltenen Erkrankungen mit Lungenbeteiligung.

die Asthmaprävalenz in Europa beträgt 4,5\% [2]. Es bleibt also festzuhalten, dass die einzelne Erkrankung zwar selten ist, dass aber die Gesamtzahl der Betroffenen mit seltenen Erkrankungen höher ist, als die mancher „Volkskrankheiten“ für sich betrachtet.

\section{Definition}

$\nabla$

In Europa sind seltene Erkrankungen über die Häufigkeit und die Beeinträchtigung der Patienten definiert. Demnach betrifft eine seltene Erkrankung weniger als 5 von 10000 Individuen. Zudem muss die Erkrankung chronisch, progredient, schwerwiegend und lebensbedrohlich sein [3].

\section{Seltene Erkrankungen in der Pneumologie \\ $\nabla$}

Etwa 80\% der seltenen Erkrankungen haben eine genetische Ursache [3], doch nicht alle sind bereits bei Geburt manifest; häufig sind mehrere Organsysteme betroffen. Bei manchen seltenen Lungenkrankheiten (s. Tab.1) treten die ersten Symptome im Erwachsenenalter auf. Einige seltene Erkrankungen sind auf Infektionen, Allergien oder Umwelteinflüsse zurückzuführen, bei anderen ist die Ursache unbekannt.

Bei diesen Erkrankungen ist die rasche Diagnose von großer Bedeutung, sodass präventive, therapeutische und ggf. rehabilitative Maßnahmen frühzeitig eingeleitet und Spätschäden vermie- den werden können. Dies trägt nachweislich zur Verbesserung der Prognose bei [4,5]. Für Erkrankungen mit einfachen diagnostischen Tests kann dies vielfach durch ein geeignetes Screening von Neugeborenen erreicht werden.

\section{Herausforderung aus der Sicht der Patienten}

Viele Patienten mit seltenen Erkrankungen haben einen langen Leidensweg bis zur korrekten Diagnosestellung hinter sich. Bei Patienten mit Alpha-1-Antitrypsinmangel (AATM) beträgt die Latenz sowohl in Deutschland als auch den USA zwischen Auftreten erster klinischer Beschwerden und der Diagnosestellung im Mittel ca. 6 Jahre und es werden hierzu durchschnittlich mehr als 3 verschiedene Ärzte kontaktiert [6]. In Extremfällen wurden bis zu 13 Ärzte konsultiert, bis die Krankheit korrekt erkannt wurde.

Bei der zystischen Fibrose beträgt die diagnostische Latenz etwa 1,2 Jahre und kann mittels etablierter Verfahren im Neugeborenenscreening auf 13 Wochen reduziert werden, also im Regelfall bereits bevor diese Säuglinge symptomatisch werden [7]. Dies ermöglicht eine frühzeitige Einleitung von prophylaktischen und therapeutischen Maßnahmen, die zur Verbesserung von Lungenfunktion, Ernährungsstatus und letztendlich auch der Prognose führen [4].

Auch bei der idiopathischen pulmonalen Fibrose (IPF) beträgt die Latenz zwischen ersten Beschwerden und Diagnosestellung im Mittel 2,2 Jahre. Auch hier besteht ein Zusammenhang zwischen verzögerter Diagnose und Mortalität [5]. 
Tab.2 Patienten mit seltenen Erkrankungen erleben folgende Hürden, die den Zugang zu einer optimalen medizinischen Versorgung erschweren [9].

Hindernis bei der Erlangung eines bestmöglichen Gesundheitsstandards

Mangelnde wissenschaftliche Kenntnis über die Erkrankung

Fehlende und oftmals falsche Diagnosestellung

Verzögerte Diagnosestellung

Mangelnde angemessene multidisziplinäre medizinische Versorgung

Unzureichende Information und Unterstützung zum Diagnosezeitpunkt

Soziale Benachteiligung

Ungleichbehandlung und Schwierigkeiten beim Zugang zu Therapie,

Rehabilitation und Versorgung

Unzufriedenheit und Vertrauensverlust bezüglich medizinischer und

sozialer Dienste

Ablehnung durch Beschäftigte im Gesundheitswesen

Daher verwundert es nicht, dass für die Patientenorganisationen eine hohe medizinische Kompetenz des behandelnden Zentrums die größte Bedeutung hat [8]. Hindernisse, die den Zugang von Patienten mit seltenen Erkrankungen zu einer optimalen medizinischen Versorgung erschweren, hat die europäische Patientenorganisation EURODIS exemplarisch zusammengefasst ( $\bullet$ Tab.2) [9].

Eine Kernforderung von Patientenorganisationen ist eine patientenzentrierte Versorgung, bei der Prävention, Diagnose und Therapie in allen Stadien eng von Forschung, Kommunikation und Qualitätssicherung begleitet werden ( $\boldsymbol{Q}$ Abb. 1) [7]. Alle unterstützenden Maßnahmen sollen den patientenrelevanten Nutzen im Focus haben. Die Forderung der Patienten bedeutet daher, dass das im Gesundheitssystem vorherrschende Sektorendenken durch die interdisziplinäre Gesamtbetrachtung der Patientenerfordernisse ersetzt werden muss. Dies setzt eine enge Kooperation aller Fachbereiche sowie eine Vernetzung der sektorenübergreifenden Betreuungsangebote (ambulante, stationäre und rehabilitative Leistungsbereiche) voraus.

Patientenorganisationen können in diesem Prozess wichtige Funktionen übernehmen und ihr Wissen über die vorhandenen Netzwerke den Patienten, ihren Familien und behandelnden Ärzten zur Verfügung stellen.

\section{Herausforderung für die Leistungserbringer}

Der Startpunkt für die Recherche nach aktuellen Informationen über eine Krankheit ist für den Arzt die Bezeichnung der Erkrankung. Doch wie ist das beste Vorgehen, wenn es zwar den Verdacht auf eine seltene Erkrankung gibt, es aber unklar ist, um welche es sich handelt? Oder wenn bei einem Syndrom mehrere
Organe betroffen sind? An wen kann man sich wenden? Für den behandelnden Arzt ist nun der Kontakt zu einem Experten von entscheidender Bedeutung. Bei Verdacht auf eine seltene Erkrankung ist das Auffinden tatsächlicher Experten auf dem Gebiet oft mit größten Schwierigkeiten verbunden.

Zur Diagnosestellung sind häufig spezielle Tests erforderlich. Die Fragen zur genauen Durchführung können meist mithilfe des zuständigen Labors geklärt werden. Doch nach dem Vorliegen des Laborbefundes muss der behandelnde Arzt die Aufklärung des Patienten über Verlauf, Prognose und Therapie der Erkrankung oft alleine durchführen. Bei den genetisch determinierten Erkrankungen kommt noch die Familienberatung hinzu, die viel Wissen über die Erkrankung, Zeit und Einfühlungsvermögen erfordert.

Aktuelle Leitlinien zu bestimmten seltenen Erkrankungen fehlen oft. Ein wesentlicher Grund für die geringe Zahl von aktuellen und Evidenz-basierten Leitlinienempfehlungen ist die spärliche Datenlage bei den seltenen Erkrankungen. Die Zahl der zugelassenen Medikamente in diesem Bereich ist trotz einer positiven Entwicklung in den letzten 10 Jahren weiterhin sehr klein [10]. Für die Patienten und die behandelnden Ärzte ist dies ein seltener Glücksfall. Die Hersteller haben im Rahmen des Zulassungsverfahrens belastbare Daten für die Sicherheit und Wirksamkeit des Medikamentes bei der untersuchten Erkrankung vorgelegt. Die Datenlage ist hier also deutlich besser als bei den Therapien der meisten anderen seltenen Erkrankungen, für die es keine zugelassenen Therapien gibt. Bestenfalls kommen manchmal für andere Indikationen zugelassenen Medikamente infrage. Der behandelnde Arzt muss dann die Erstattungsfrage klären und geht möglicherweise das Risiko eines Regresses ein. Beim „Off Label“ Einsatz von Medikamenten besteht darüber hinaus eine erweiterte Aufklärungs- und Dokumentationspflicht gegenüber dem Patienten.

\section{Herausforderung für das Gesundheitssystem}

Das Gesundheitssystem hat die Aufgabe, Strukturen und Ressourcen für die Betreuung von Patienten vorzuhalten. Diese Aufgabe ist aufgrund fehlender Informationen über die seltenen Erkrankungen nur schwer zu erfüllen.

In Deutschland sind nur unzureichende Erkenntnisse über die Qualität und den Umfang der Versorgung von Patienten mit seltenen Erkrankungen verfügbar [8]. Es fehlen detaillierte Kenntnisse über die Inanspruchnahme von Leistungen zu bestimmten seltenen Erkrankungen. Diese Daten könnten über eine Analyse der ICD-Kodierungen im Prinzip erhoben werden, doch existiert

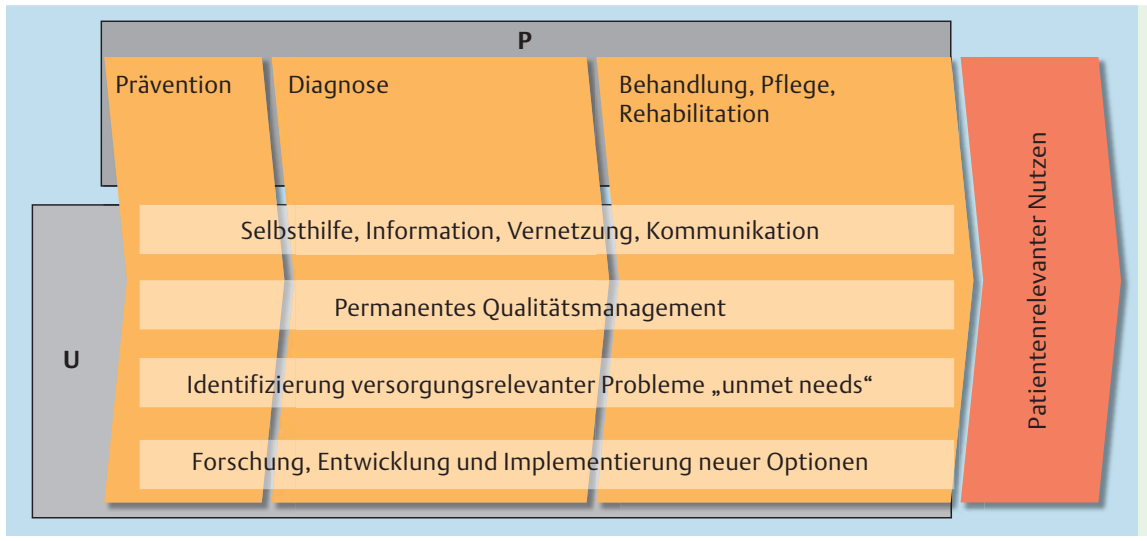

Abb. 1 Die patientenzentrierte Versorgung von Betroffenen mit seltenen Erkrankungen gliedert sich in primäre medizinische Aktivitäten $(\mathrm{P})$ und in unterstützende Aktivitäten, die idealerweise mit dem gesamten medizinischen Komplex vernetzt sind und diesen begleiten (aus [7]). 
bislang lediglich für ungefähr 250 der etwa 6000 seltenen Erkrankungen eine eindeutige ICD-10-Klassifikation [1]. Daher entwickeln Arbeitsgruppen Vorlagen für die Erweiterung der ICDKlassifikation durch die Weltgesundheitsorganisation (WHO). Insbesondere von den Kostenträgern werden die hohen Preise der Medikamente für seltene Erkrankungen mit Sorge gesehen. Die neu zugelassenen Medikamente sind zumeist im oberen Preissegment zu finden, die Anzahl der damit behandelten Patienten ist jedoch bei seltenen Erkrankungen naturgemäß gering. Insgesamt betragen die Kosten für Orphan Drugs weniger als $1 \%$ der Gesundheitsausgaben in Europa [11].

\section{Lösungsansätze zur Verbesserung der Versorgung bei seltenen Erkrankungen \\ $\nabla$}

Die Entwicklung von neuen Medikamenten ist teuer und aufwendig. Bis eine neu entdeckte Substanz die Zulassung der Arzneimittelbehörden erhält, werden 5000 bis 10000 Substanzen auf ihre Wirksamkeit untersucht. Von diesen erreichen 250 das Stadium der prä-klinischen Forschung, und nur 5 werden bei Patienten in klinischen Studien untersucht [12]. Die Entwicklung eines neuen Medikamentes kostet über 800 Mio US-\$ (Stand 2003) $[12,13]$, und die Kosten steigen weiter um ca. $10 \%$ pro Jahr. Damit droht die Entwicklung neuer Medikamente für die entwickelnden Pharmafirmen unerschwinglich zu werden [13].

Auch die Europäische Zulassungsbehörde (European Medicine Agency, EMA) hat erkannt, dass dieses wirtschaftliche Risiko entwicklungshemmend wirkt [14]. Um die Entwicklung von Medikamenten für seltene Erkrankungen („Orphan Drugs“) zu fördern, wurden Anreize für Arzneimittelhersteller geschaffen. So erhalten in Europa zugelassene Orphan Drugs eine Marktexklusivität von 10 Jahren [14]. Auch dank dieser Maßnahmen ist die Zahl zugelassener Orphan Drugs in den letzten 10 Jahren angestiegen [15].

Ein weiteres politisches Ziel ist die verbesserte integrierte Versorgung bei seltenen Erkrankungen ( Tab.3) [1]. Im Auftrag des Bundesministeriums für Gesundheit (BMG) wurde eine Bestandsaufnahme durchgeführt [8]. In der Folge hat im März 2010 das Nationale Aktionsbündnis für Menschen mit seltenen Erkrankungen (NAMSE), ein Zusammenschluss zwischen dem BMG, dem Bundesministerium für Bildung und Forschung (BMBF) und dem Achse e.V. (Allianz Chronischer Seltener Erkrankungen) mit 23 weiteren Bündnispartnern die Arbeit aufgenommen. Das NAMSE hat die Aufgabe, Maßnahmen zu entwickeln, um die Diagnosestellung zu beschleunigen, Fachzentren zu schaffen, Experten zu vernetzen und die Forschung zu verbessern. Die Ergebnisse münden in einen nationalen Aktionsplan, der dann als Basis für strukturelle Änderungen im Gesundheitswesen dienen soll.

Tab. 3 EU-Ratsempfehlung [1] an die Länder der EU, um die Versorgung von Patienten mit seltenen Erkrankungen zu verbessern.

1. Nationale Aktionspläne/Strategien für seltene Erkrankungen bis 2013

2. Definition, Kodierung und Bestandsaufnahme seltener Erkrankungen

3. Erforschung seltener Erkrankungen

4. Fachzentren und europäische Referenznetze für seltenen Krankheiten bis 2013

5. Förderung des Wissensaustausches/Zusammenführung des Fachwissens über seltene Krankheiten auf europäischer Ebene 6. Stärkung der Selbsthilfe/Mitbestimmung von Patientenverbänden 7. Sicherung der Nachhaltigkeit der vorgeschlagenen Maßnahmen

\section{Vorhandene Strukturen und Hilfsmittel}

OrphaNet ist eine Online-Ressource mit einer umfassenden ständig aktualisierten Datenbank zu den einzelnen seltenen Erkrankungen. Neben der Beschreibung vieler Erkrankungen finden sich hier auch Links zu klinischen Studien, zugelassenen Medikamenten, Expertenzentren und Patientenorganisationen. Von den etwa 6000 bekannten seltenen Erkrankungen sind derzeit 1330 mit erklärenden Artikeln in der Datenbank aufgenommen (OrphaNet, Zugriff 13.03.2012). Diese Diskrepanz verdeutlicht noch einmal, wie schwierig die Sammlung von verlässlichen Informationen für manche seltene Erkrankung ist.

Orphanet ist vernetzt mit der OMIM-Datenbank (Online Mendelian Inheritance in Man) in der Informationen über die bekannten Mutationen, Diagnosemöglichkeiten und aktuelle wissenschaftliche Entwicklungen aufgelistet sind.

\section{Bedeutung von Kompetenzzentren}

Für die Betreuung von Patienten mit seltenen Erkrankungen sind nationale Kompetenzzentren ganz besonders wertvoll. In diesen zumeist universitären Zentren können Patientenversorgung, Grundlagenforschung und klinische Studien an einem Ort gebündelt werden. Die Vernetzung im Sinne der sektorenübergreifenden patientenzentrierten Versorgung sowie ein interdisziplinärer Austausch sind hier am ehesten möglich. Die Expertenzentren können bei der Diagnose, Einleitung von spezifischen Therapien sowie der Verlaufskontrolle behilflich sein. Sie können die Beratung von Patienten und Angehörigen mit seltenen Erkrankungen übernehmen und unterstützen somit den behandelnden Arzt. Die Auswirkung bereits bestehender medizinischer Netzwerke auf die Patientenversorgung wird als hoch oder sehr hoch eingeschätzt [8]. Aus den genannten Gründen kommt dem Aufbau bzw. der Stärkung von Referenzzentren für seltene Erkrankungen eine besondere Bedeutung zu.

\section{Bedeutung von Registern}

Bei vielen seltenen Erkrankungen können Register durch die Zusammenarbeit von nationalen und internationalen Zentren in überschaubarer Zeit eine große Zahl von Patienten einschließen und nachverfolgen.

Mittels gut strukturierter Fragebögen können prospektiv Daten über den natürlichen Verlauf der Erkrankung, die Lebensqualität, Mortalität sowie Behandlungs- und Wirksamkeitsdaten erhoben werden [16-18]. Durch professionelle Organisation, definierte Case Report Forms (CRFs) und Monitorierung der Daten kann auch in Registern eine exzellente Datenqualität mit vollständigen Datensätzen erreicht werden. Die Quote der fehlenden Daten ist z.T. extrem gering [19]. Wie bei klinischen Studien werden die teilnehmenden Zentren z.T. auditiert und die Patientendaten monitoriert $[18,19]$. So können Daten über längerfristige Verläufe der Erkrankung sowie Häufigkeiten verschiedener Therapieformen einschließlich Kombinationstherapien und Therapiewechsel erfasst werden, welche die klinische Versorgung widerspiegeln $[18,19]$. Eine Auswertung von prognostischen Parametern auf Zentrumsebene ermöglicht einen Vergleich mit anderen teilnehmenden Zentren, sodass erfolgreiche klinische Betreuungsmodelle identifiziert und auf die anderen Zentren übertragen werden können [19].

Trotz dieser erfolgreichen Beispiele für die Etablierung von Registern ist deren Gesamtzahl gemessen an der Zahl der seltenen Erkrankungen weiter sehr gering. Krankheitsbezogene Register könnten aber auch wertvolle Informationen über Epidemiologie und Verlauf der bislang nicht untersuchten seltenen Erkrankun- 
gen geben. Eine finanzielle Unterstützung von Registern mit dem Ziel der Professionalisierung kann von wesentlicher Bedeutung sein. Hierzu müssen neue Wege für die Organisation und Finanzierung z. B. durch Public-Private Partnerships gefunden werden.

\section{Förderung zur Entwicklung von Orphan Drugs}

In Bereichen, die für Pharmafirmen nicht attraktiv erscheinen, muss die öffentliche Hand auch Forschung zur Entwicklung neuer Therapieansätze finanziell unterstützen, wie das bereits vom amerikanischen National Institute of Health (NIH) exemplarisch vorgemacht wird.

Das amerikanische NHLBI führt seit 2009 eine 3-armige klinische Studie zur Wirksamkeit von häufig eingesetzten Therapien bei Patienten mit IPF (PANTHER Study) durch (NCT00650091, http://clinicaltrials.gov). Kürzlich wurde der Arm für eine Kombinationstherapie (Prednisolon, Azathioprin und N-Acetylcystein (NAC)) wegen einer erhöhten Mortalität im Vergleich zu Placebo geschlossen. Die beiden verbleibenden Studienarme (NAC-Monotherapie und Placebo) werden unverändert weiter geführt [20]. Die eingesetzten Medikamente sind alle bereits seit langem für andere Indikationen auf dem Markt verfügbar, billige Generika sind ebenfalls zugelassen. Daher ist das Interesse der forschenden Pharmafirmen gering, klinische Studien mit diesen Substanzen durchzuführen und auf eine Zulassung für eine seltene Erkrankung zu hoffen. Diese Studie wäre daher ohne öffentliche Förderung nicht möglich gewesen.

In Europa sind über die Framework-Programme der EU-Fördergelder für seltene Erkrankungen verfügbar, der Schwerpunkt der geförderten Projekte variiert jedoch je nach Ausschreibungszyklus.

\section{Ausblick \\ $\nabla$}

Das Thema der Versorgung von Patienten mit seltenen Erkrankungen hat in den letzten Jahren viel Aufmerksamkeit erhalten. Eine Reihe von politischen Initiativen sind zu diesem Zweck in Europa gestartet worden. In der Folge werden politische Vorgaben aus der EU auf Länderebene zur Umsetzung gebracht werden und so in die Strukturen für die Versorgung von seltenen Erkrankungen eingreifen. Dieser Prozess schließt die Fachgesellschaften bislang nicht ein.

Um eine verbesserte Versorgung von Betroffenen zu erreichen, sind eine stärkere Vernetzung der bereits vorhandenen Expertise und die Intensivierung von Informationsaustausch erforderlich. Des Weiteren sollten Fachzentren aufgebaut werden, die sich schwerpunktmäßig der interdisziplinären Betreuung von Patienten mit seltenen Erkrankungen widmen. Hier ist auch ein vermehrtes Engagement der Fachgesellschaften wünschenswert.

\section{Interessenkonflikt}

$\nabla$

Die Autoren geben an, dass kein Interessenkonflikt besteht.

\section{Literatur}

1 Council of the European Union. Council Recommendation on an Action in the Field of Rare Diseases. Official Journal of the European Union 2009: C 151/02 7-10

2 Burney P, Chinn S, Jarvis D et al. Variations in the prevalence of respiratory symptoms, self-reported asthma attacks, and use of asthma medication in the European Community Respiratory Health Survey (ECRHS). Eur Respir J 1996; 9: 687-695

3 EMEA. Orphan drugs and rare diseases at a glance. Doc. Ref. EMEA/ 290072/2007 3-7-2007

4 Zemanick ET, Harris JK, Conway $S$ et al. Measuring and improving respiratory outcomes in cystic fibrosis lung disease: opportunities and challenges to therapy. J Cyst Fibros 2010; 9: 1-16

5 Lamas DJ, Kawut SM, Bagiella $E$ et al. Delayed access and survival in idiopathic pulmonary fibrosis: a cohort study. Am J Respir Crit Care Med 2011; 184: $842-847$

6 Köhnlein T, Janciauskiene S, Welte T. Diagnostic delay and clinical modifiers in alpha-1 antitrypsin deficiency. Ther Adv Respir Dis 2010; 4: 279-287

7 Reimann A, Bend J, Dembski B. Patientenzentrierte Versorgung bei seltenen Erkrankungen: Perspektive von Patientenorganisationen. Bundesgesundheitsblatt Gesundheitsforschung Gesundheitsschutz 2007; 50: $1484-1493$

8 Eidt $D$, Frank M, Reimann A et al. Maßnahmen zur Verbesserung der gesundheitlichen Situation von Menschen mit Seltenen Erkrankungen in Deutschland. 2009: 1 - 319

9 EURORDIS. The Voice of 12,000 patients. 2009. ISBN: 978-29530318-1-2

10 Orphanet Report Series. Lists of Orphan Drugs in Europe 2012. http:// www.orpha.net/orphacom/cahiers/docs/DE/Verzeichnis_der_in_Europa_zugelassenen_Orphan_Drugs.pdf

11 Tambuyzer E. Rare diseases, orphan drugs and their regulation: questions and misconceptions. Nat Rev Drug Discov 2010; 9: 921 - 929

12 DiMasi JA, Hansen RW, Grabowski HG. The price of innovation: new estimates of drug development costs. J Health Econ 2003; 22: 151 - 185

13 Rawlins MD. Cutting the cost of drug development? Nat Rev Drug Discov 2004; 3: 360-364

14 Council of the European Union. Regulation No 141/2000 on Orphan Medicinal Products. Official Journal of the European Communities 2000: $\mathrm{L} 18 / 1$

15 Aymé S, Hivert V. Orphanet Report Series. Report on rare disease research, its determinants in Europe and the way forward 2011. http:// asso.orpha.net/RDPlatform/upload/file/RDPlatform_final_report.pdf

16 The Alpha-1-Antitrypsin Deficiency Registry Study Group. Survival and FEV1 decline in individuals with severe deficiency of alpha1-antitrypsin. Am J Respir Crit Care Med 1998; 158: 49-59

17 Seersholm N, Wencker M, Banik $N$ et al. Wissenschaftliche Arbeitsgemeinschaft zur Therapie von Lungenerkrankungen (WATL) alpha1-AT study group. Does alpha1-antitrypsin augmentation therapy slow the annual decline in FEV1 in patients with severe hereditary alpha1-antitrypsin deficiency? Eur Respir J 1997; 10: 2260-2263

18 Pittrow D, Ghofrani HA, Opitz CF et al. Internationales, prospektives Register zur Erfassung der Initial- und Dauertherapie von Patienten mit pulmonaler Hypertonie (CompERA-XL). Dtsch Med Wochenschr 2009; 134: $173-175$

19 Stern $M$, Wiedemann $B$, Wenzlaff $P$. From registry to quality management: the German Cystic Fibrosis Quality Assessment project 19952006. Eur Respir J 2008; 31: 29-35

20 NHLBI press release. Commonly used three-drug regimen for idiopathic pulmonary fibrosis found harmful 21-10-2012. http://public. nhlbi.nih.gov/newsroom/home/GetPressRelease.aspx?id=2812

21 Rolke M, Rumpf J, Kroidl R et al. Neue epidemiologische Daten zur exogen-allergischen Alveolitis in Deutschland. Allergologie 2006; 29: 439-442

22 Orphanet Report Series. Prevalence of rare diseases: Bibliographic data 2010; Number 21-27. http://www.orpha.net/orphacom/cahiers/docs/ GB/Prevalence_of_rare_diseases_by_decreasing_prevalence_or_cases. pdf 